\title{
A Divide and Conquer Strategy against the Covid-19 Pandemic?!
}

\author{
Patrick Mangat*
}

Dated: 2020-05-05

\begin{abstract}
The concern about (socio-)economic consequences of collective lockdowns in the Covid19 pandemic calls for alternative strategies. We consider a divide and conquer strategy in which a high risk group (HRG) is put on strict isolation, whereas the remainder of the population is exposed to the virus, building up immunity against Covid-19. The question is whether this strategy may suppress the effective reproduction number below the critical value of $\mathcal{R}_{\text {eff }}<1$ without further lockdown once the HRG is released from isolation. While this proposal appears already rather academic, we show that $\mathcal{R}_{\text {eff }}<1$ can only be obtained provided that the HRG is less than $\sim 20-30 \%$ of the total population. Hence, this strategy is likely to fail in countries with a HRG larger than the given upper bound. In addition, we argue that the maximum infection rate occurring in this strategy is likely to exceed realistic capacities of most health care systems. While the conclusion is rather negative in this regard, we emphasise that the strategy of stopping the curve at an early stage of the Covid-19 pandemic has a chance to work out. The required duration of the lockdown is estimated to be $\tau \sim 14$ days $/\left(1-\mathcal{R}_{\text {eff }}\right.$ ) (up to some order one factor) for $\mathcal{R}_{\text {eff }}<1$, provided a systematic tracing strategy of new infections exists for the subsequent relaxation phase. In this context we also argue why $\mathcal{R}_{\text {eff remains the crucial parameter }}$ which needs to be accurately monitored and controlled.
\end{abstract}

Key words - Epidemiology, pandemics, Covid-19, quarantine, SIR-model, differential equations.

\section{Introduction}

Most countries have been locked down for more than six weeks already. The goal of the lockdown strategy is to avoid a collapse of the health care systems. However, this goes at the cost of significant economic decline including growing debts and unemployment (for studies on (socio-)economic impacts see e.g. [1-7]). While the Covid-19 pandemic directly threatens our health, the link between economic growth and our health is rather implicit and non-trivial. The lockdown particularly protects the so-called high risk group (HRG) [8-10], whereas the economic consequences will affect the whole population. Consequently, in Germany (and most likely in other countries as well) doubts have been raised whether the current mitigation measures are proportionate.

In particular, it has been proposed whether it may suffice to impose isolations only for the HRG, while the rest of the population is exposed to the virus (various discussions can be found in German newspapers such as Spiegel Online). The hope behind this proposal is that the decline of the economic growth is minimised while the health care system is kept stable (because the non-HRG is less likely to require intensive treatment), see e.g. [1] for related work in this direction. Apart from various practical issues with this proposal, this strategy makes only sense if the effective reproduction number eventually drops below $\mathcal{R}_{\text {eff }}<1$ once

*E-mail: patrick.mangat@gmail.com; Address: Dr. Patrick Mangat, Department of Electrical Engineering, Mannheim University of Applied Science, Paul-Wittsack-Straße 10, 68163 Mannheim, Germany

NOTE: This preprint reports new research that has not been certified by peer review and should not be used to guide clinical practice. 
the HRG can be released from the isolation. Of course, the hope is that $\mathcal{R}_{\text {eff }}<1$ is reached purely due to the immunity of the non-HRG. This would mean that the Covid-19 pandemic is in control even without further measures afterwards.

We call this the divide and conquer strategy, because the population is divided into a HRG and a non-HRG. Using the SIR-model [11] we prove that the divide and conquer strategy requires the HRG to be less than approximately $20-30 \%$ of the whole population. Hence, at least in Germany this strategy will most likely be unsuccessful. In addition, we argue that realistic capacities of the German health care system are likely to be exceeded due to the infections of the non-HRG in this strategy. We use the SIR-model, because it captures the relevant mechanisms of a pandemic despite its simplicity.

This note is organised as follows. We briefly recall the basics of the SIR-model including an application to Covid-19 and the flatten the curve strategy. Afterwards we apply the SIR-model to the divide and conquer strategy. Finally, we summarise the importance of the reproduction number in this crisis and propose a simple formula to estimate how long one needs to keep $\mathcal{R}_{\text {eff }}<1$ until a lockdown can be relaxed.

\section{A primer on the SIR-model}

This section recalls the basics of the SIR-model (following [12-14]) and briefly demonstrates the dynamics in the Covid-19 pandemic as well as the problem with flattening the curve in this context. Readers who are familiar with this model can skip to the next section.

\section{$2.1 \quad$ The SIR-model}

The SIR-model divides the population into susceptible $(S)$, infectious $(I)$ and recovered $(R)$ people. This is the origin of the name of the model developed in [11]. The total population $N$ is then given by

$$
N=S+I+R \text {. }
$$

The SIR-model assumes constant population, i.e. no deaths, births and migration. An infectious person will infect a susceptible person at a transmission rate $\beta$, and will recover at a recovery rate $\gamma$. Any recovered person is assumed to be immune against the disease forever.

The system of differential equations then reads

$$
\begin{aligned}
\dot{S} & =-\beta S I, \\
\dot{I} & =\beta S I-\gamma I, \\
\dot{R} & =\gamma I,
\end{aligned}
$$

where $\dot{S}=d S / d t$ (derivative of $S$ with respect to time $t$ ) and likewise for $\dot{I}$ and $\dot{R}$. By adding up those three differential equations one can easily see that $\dot{N}=0$, i.e. the total number of population is indeed conserved.

A crucial parameter is the basic reproduction number $\mathcal{R}_{0}$, which can be written at $t=0$ as

$$
\mathcal{R}_{0}=\frac{\beta}{\gamma} S(0) .
$$

The basic reproduction number tells us to how many people the disease is transmitted on average due to one infectious individual. To see where (2.5) comes from, we suppose that at $t=0$ we have $I(0)=1$. The probability of this infectious person to remain infected at time $t$ 
is $p(t)=e^{-\gamma t}$. Hence, the expectation value for the number of people this single person will infect, is given by

$$
\beta S(0) \int_{0}^{\infty} d t p(t)=\frac{\beta}{\gamma} S(0)
$$

Given $\mathcal{R}_{0}$, the effective reproduction number $\mathcal{R}_{\text {eff }}=\mathcal{R}(t)$ is

$$
\mathcal{R}(t)=\frac{\beta}{\gamma} S(t)=\mathcal{R}_{0} \frac{S(t)}{S(0)}
$$

Hence, as the pandemic is progressing, one infectious person will infect fewer and fewer people on average, because more and more people recover in the course of this pandemic.

Therefore, the effective reproduction number decreases automatically and, eventually, it will drop below the critical value of $\mathcal{R}_{\text {eff }}=1$. This is when herd immunity is reached.

To understand this in detail, we rewrite $(2.3)$ in terms of $\mathcal{R}(t)$ :

$$
\dot{I}(t)=\gamma(\mathcal{R}(t)-1) I(t) .
$$

By linearising the differential equations one can rigorously prove that an epidemic occurs if and only if $\mathcal{R}_{0}>1$. A less rigorous argument is the following. Initially, $\mathcal{R}(t) \simeq \mathcal{R}_{0}$ because $S(t) \simeq S(0) \simeq N$ initially in a large population with only few infectious and recovered people. Then, (2.8) immediately implies an exponential growth of infectious people, namely

$$
I(t) \simeq I(0) e^{\gamma\left(\mathcal{R}_{0}-1\right) t},
$$

at the beginning of the epidemic. For $\mathcal{R}_{0}>1$ the disease is spreading exponentially, whereas for $\mathcal{R}_{0}<1$ the recovery is faster than the spread leading to exponential suppression. At the critical value of $\mathcal{R}_{0}=1$ the number of new infections is compensated by the number of recovered people (per time interval).

But once the number of immune people increases to a significant amount, the $I$-curve deviates from an exponential function, and eventually reaches its maximum, at which $\mathcal{R}(t)=$ 1. This is when herd immunity is reached, because from then on $\dot{I}(t)<0$ until $I(t) \rightarrow 0$.

At herd immunity the proportion of people that have been infected is given by

$$
q=1-\frac{1}{\mathcal{R}_{0}} .
$$

To see this, note that at $t=t_{\mathrm{H}}$ (time of herd immunity) we have

$$
1=\mathcal{R}\left(t_{\mathrm{H}}\right)=\mathcal{R}_{0} \frac{S\left(t_{\mathrm{H}}\right)}{S(0)} \simeq \mathcal{R}_{0} \frac{S\left(t_{\mathrm{H}}\right)}{N} .
$$

By definition, $q \equiv 1-S\left(t_{\mathrm{H}}\right) / N$ and the assertion (2.10) follows.

Despite herd immunity there will still be further new infections at a rate

$$
\dot{I}_{\text {new }}(t)=\gamma \mathcal{R}(t) I(t),
$$

i.e. at $t \gg t_{\mathrm{H}}$ a significantly larger fraction than $q$ will have had contact with the disease.

Let

$$
\alpha \equiv \lim _{t \rightarrow \infty} \frac{R(t)}{S(0)}
$$

be the fraction of people who will eventually get into contact with the virus in the course of the pandemic (for $S(0) \simeq N$ this represents the fraction of the whole population). We can rearrange (2.2) and (2.4) to

$$
\frac{d S}{d R}=-\mathcal{R}_{0} \frac{S}{S(0)} .
$$


Solving this differential equation results in

$$
1-\alpha-e^{-\mathcal{R}_{0} \alpha}=0
$$

For $\alpha \neq 0$ this equation has only a solution in terms of the Lambert $W$ function. We observe that $\alpha=\alpha\left(\mathcal{R}_{0}\right)$, i.e. the quantity $\alpha$ is purely determined by the reproduction number. This equation will be important in Section 3.

Another important equation regards the maximum number of people that are simultaneously infected, $I_{\max }$, reached at herd immunity. Solving $\dot{I}\left(t_{\mathrm{H}}\right)=0$ implies $S\left(t_{\mathrm{H}}\right)=\gamma / \beta$. Furthermore, (2.2) and (2.3) imply

$$
\frac{d I}{d S}=\frac{\gamma}{\beta S}-1
$$

The general solution is

$$
I(S)=\frac{\gamma}{\beta} \ln \left(\frac{S}{S(0)}\right)+I(0)+S(0)-S
$$

At $t=t_{\mathrm{H}}$ we then have

$$
I_{\max }=S(0) \frac{\mathcal{R}_{0}-\ln \left(\mathcal{R}_{0}\right)-1}{\mathcal{R}_{0}}+I(0)
$$

But more important than this number is the maximum of the daily new infections, which should be below the health care capacities. The condition for the maximum of new infections reads

$$
0=\ddot{I}_{\text {new }}=\beta \dot{S} I+\beta S \dot{I}
$$

which yields

$$
S=\frac{\gamma}{\beta}+I
$$

This can be inserted into (2.17), yielding a complicated equation in $I$. But in the limit $\mathcal{R}_{0} \gg I / S(0)$ we obtain

$$
I \simeq S(0)\left(1-\frac{\ln \left(\mathcal{R}_{0}\right)}{\mathcal{R}_{0}}-\frac{1}{\mathcal{R}_{0}}\right)
$$

The limit $\mathcal{R}_{0} \gg I / S(0)$ is roughly satisfied for $\mathcal{R}_{0} \lesssim 2$. Hence the maximum number of new infections in this limit is

$$
\left(\frac{d I_{\text {new }}}{d t}\right)_{\max } \simeq \gamma S(0)\left(\mathcal{R}_{0}-\ln \left(\mathcal{R}_{0}\right)\right)\left(1-\frac{\ln \left(\mathcal{R}_{0}\right)}{\mathcal{R}_{0}}-\frac{1}{\mathcal{R}_{0}}\right) \quad \text { for } 1<\mathcal{R}_{0}<2 .
$$

If $\mathcal{R}_{0}>2$ then the result becomes imprecise and, therefore, requires either the inclusion of higher order corrections in $I /\left(\mathcal{R}_{0} S(0)\right)$ or a numerical approach. This number of new daily infections must be smaller than the capacity of the health care system. (Of course, this capacity is non-trivial to estimate.)

\subsection{Application to the Covid-19 pandemic}

We apply the SIR-model to the Covid-19 pandemic in Germany. The intention is merely to illustrate the underlying dynamics rather than making any predictions. In the past months a lot of effort has been put into mathematical modelling of the Covid-19 pandemic, see e.g. [1528] for an extensive, yet incomplete, list. 

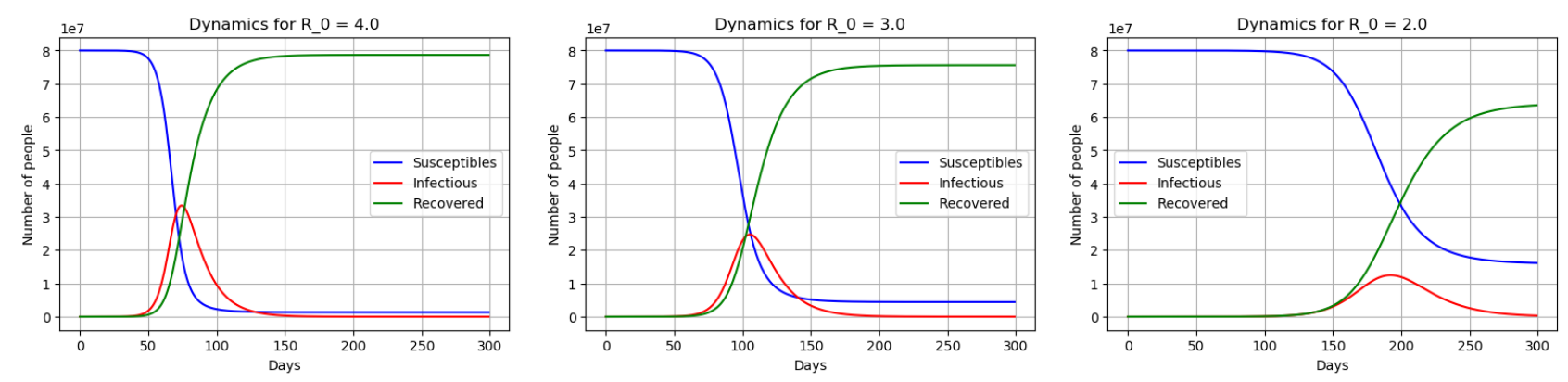

Figure 1: These plots show the dynamics of the Covid-19 pandemic for reproduction numbers $\mathcal{R}_{0} \in\{2.0 ; 3.0 ; 4.0\}$. The number of susceptible (blue), infectious (red) and recovered (green) people is plotted against time (in days). It is emphasised that these plots are not suitable for predictions. Instead they merely reveal the underlying dynamics of the SIR-model.

First of all, we notice that the SIR-model is approximately suitable to describe the Covid19 pandemic, because to present knowledge a recovered person is immune against reinfections for some significant time at least. ${ }^{1}$ However, some infections lead to death. But technically, dead people can be treated like recovered people in the SIR-model. Therefore, we do not need to modify the model for the purpose of this note. In more realistic models of epidemiology one should also include the status "exposed", in which a person got infected without being infectious yet. This is accounted for in the SEIR-model. Many more complications can be introduced, such as asymptomatic cases etc., see e.g. [16] for a sophisticated model tailored to describe Covid-19 pandemic.

In the SIR-model of Covid-19 we can set the recovery rate $\gamma=1 / 14$ day $^{-1} \simeq 0.071$ day $^{-1}$, because typically it requires 14 days to recover from Covid-19.

Estimations of $\mathcal{R}_{0}$ range from $1.4 \lesssim \mathcal{R}_{0} \lesssim 5.7$, see [29-32] and references therein. A precise value does not only depend on the virus itself but also on various external conditions such as hygienic standards, people's behaviour, density of population (see [33]), or possibly even climate (see e.g. [34]). For the first two weeks of March we can estimate for Germany [35]:

$$
\mathcal{R}_{0}=1+\frac{1}{\left(1 / 14 \text { day }^{-1}\right) \cdot 14 \text { days }} \cdot \ln \left(\frac{I(14)}{I(0)}\right)=1+\ln \left(\frac{4530}{114}\right) \simeq 4.7 .
$$

Of course, this estimation is problematic, because neither the number of tests nor the number of undetected cases is known (a transparent approach has been presented in [22]). Since the testing has become more intense in this period, the above is likely to overestimate $\mathcal{R}_{0}$. Nevertheless our estimate is still consistent with the figures computed in [16].

In Figure 1 the underlying dynamics of the SIR-model is shown. The eqs. (2.2) to (2.4) have been integrated numerically via the Euler method with initial conditions $S(0)=80 \cdot 10^{6}$, $I(0)=114$ and $R(0)=0$. We observe that smaller $\mathcal{R}_{0}$ suppresses the peak $I_{\max }$ (see also (2.18) for an analytical expression), and also shifts the time of herd immunity, $t_{\mathrm{H}}$, further into the future. In particular, for a Covid-19 pandemic without any mitigation attempts we can estimate 2 months $\lesssim t_{\mathrm{H}} \lesssim 6$ months. This estimation intends to give an idea about time scales of a pandemic. Moreover, unsurprisingly, the plateau of the $R$-curve decreases for smaller $\mathcal{R}_{0}$. Using (2.15) we obtain $\alpha(2.0) \simeq 0.80, \alpha(3.0)=0.94$ and $\alpha(4.0)=0.98$. Hence, without any measures between $80 \%-98 \%$ of the population will have had contact with the virus at the end of the pandemic. Note that those figures are not in contrast to the often quoted $60 \%-70 \%$

\footnotetext{
${ }^{1}$ Once immunity gets lost the SIR-model can be easily extended to the SIRS-model (see e.g. [12]).
} 
of infections. Those numbers refer to the moment of herd immunity. Indeed, from (2.10) we get $q(2.0)=50 \%, q(3.0)=67 \%$ and $q(4.0)=75 \%$.

Furthermore we observe that even in the case of $\mathcal{R}_{0}=2$ more that $13 \%$ of the population will be infectious at the peak of the pandemic. This is clearly unacceptable given the relatively significant case fatality rates. The situation therefore triggered many ideas how to mitigate worst case scenarios within this pandemic (see e.g. $[1 ; 2 ; 17 ; 19 ; 33 ; 36-45])$. Rather intuitive strategies that had been suggested are the concepts of flattening the curve $\left(\mathcal{R}_{\text {eff }} \gtrsim 1\right)$ and stopping the curve $\left(\mathcal{R}_{\mathrm{eff}}<1\right)$.

\subsection{Flattening the curve and fine-tuning in the Covid-19 pandemic}

In this section we point out a problem with flattening the curve. The curve that needs to be flattened is the $I$-curve, or, more strictly speaking, the rate of new infections curve, $\dot{I}_{\text {new }}(t)=\beta S(t) I(t)$. The latter curve will reach its peak before reaching herd immunity. This curve must be flattened in a way such that the peak will be below some upper bound $C$ due to capacities of the health care system. It must be ensured that

$$
\dot{I}_{\text {new }}(t) \leqslant C \quad \text { at any time } .
$$

A simplified estimation of $C$ may be as follows. Let $n_{\text {bed }}=30 \cdot 10^{3}$ be the number of available beds in intensive care units of hospitals to treat severe cases at an average duration of $\Delta t=10$ days. The probability of any random infectious person to require intensive care is assumed to be $p=5 \%$. Then,

$$
C \sim \frac{n_{\text {bed }}}{p \Delta t}=60 \cdot 10^{3} \text { day }^{-1}
$$

This figure can only be seen as a very rough estimate, because each of its variables is just an estimate. In addition, any constraint regarding health care staff or further necessary surgery equipment has been ignored. Nevertheless, this number of $C \sim 60 \cdot 10^{3}$ day $^{-1}$ suffices to point out the problem with the concept of flattening the curve below $C$.

To see this we plot $\dot{I}_{\text {new }}(t)$ for the parameter range $\mathcal{R}_{\text {eff }}=1.1$ to 1.3 . We use the notation $\mathcal{R}_{\text {eff }}$ instead of $\mathcal{R}_{0}$ because the rather small numbers are assumed to arise from some measures (like social distancing). The plot can be easily obtained numerically by computing $I(t+1)-$ $I(t)$. The result is shown in Figure 2. We chose initial conditions as of 2020-05-03 without aiming at guessing further unknown cases. The results shown are not meant to be a prediction of the future.

We find that already the first decimal place of $\mathcal{R}_{\text {eff }}$ has strong influence on the maximum height of the curve $\dot{I}_{\text {new }}$ (see (2.22) for an approximative formula). A jump from $\mathcal{R}_{\text {eff }}$ from 1.1 to 1.3 suffices to increase the peak of daily new infections by a factor larger than 6 . In particular we see that $\mathcal{R}_{\text {eff }}<1.2$ is needed to remain below the bound of $60 \cdot 10^{3}$ infections per day. Therefore, the parameter range of the effective reproduction number in flattening the curve is $1<\mathcal{R}_{\text {eff }}<1.2$, and hence very tiny. We call this requirement fine-tuning. The weaker the health care system the stronger the fine-tuning needs to be. Given the error bars in measuring $\mathcal{R}_{\text {eff }}$ (see [16]) it seems quite challenging to keep control of the pandemic within this strategy. In particular, the precise impact of different measures on $\mathcal{R}_{\text {eff }}$ is unknown at present. Furthermore, the strategy flatten the curve seems to require a duration of larger than one year! Consequently, flattening the curve seems not be the way to go in the Covid-19 pandemic. 


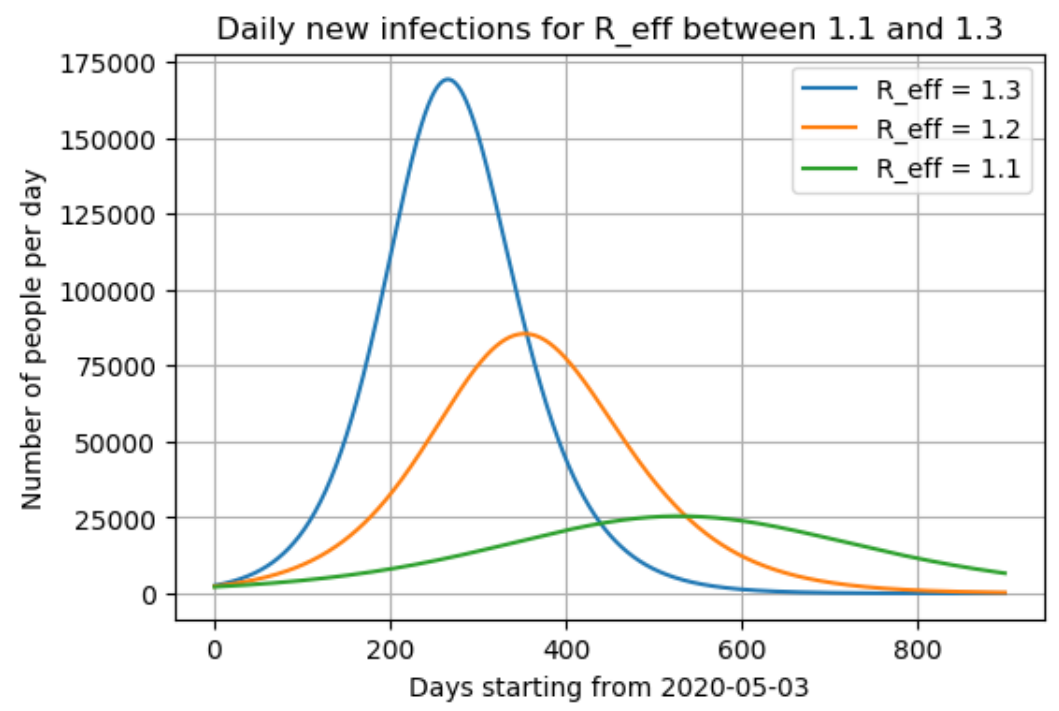

Figure 2: This plot shows the expected daily infections for the Covid-19 pandemic in Germany

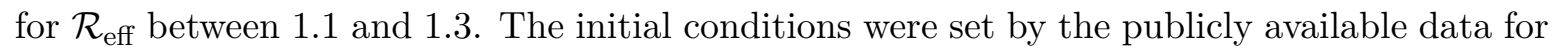
2020-05-03 [35].

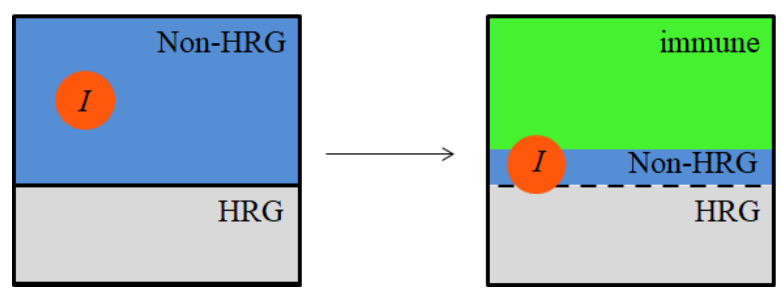

Figure 3: Illustration of the divide and conquer scenario. The whole population is initially split into a high risk group (HRG) and a non-HRG. Infectious individuals are assumed to exist only among the non-HRG, while the HRG is strictly isolated. As time evolves most of the non-HRG become immune. The HRG is released from the isolation if $\mathcal{R}_{\text {eff }}<1$ can be ensured due to the large fraction of immune people.

\section{The divide and conquer strategy}

Since the measures against the Covid-19 pandemic particularly protect the high risk group (HRG) the following divide and conquer strategy may appear worth considering: Only the HRG is strictly isolated while no significant measures are imposed on the non-HRG. We assume that infectious people only exist among the non-HRG. This situation is illustrated on the left hand side of Figure 3. Due to the dynamics explained in Section 2 most of the initially susceptible people become immune. If the proportion of immune people is large enough it is conceivable that $\mathcal{R}_{\text {eff }}<1$ even upon releasing the HRG from the isolation (depicted on the right hand side of Figure 3).

One may hope that such a strategy can keep the economy alive without risking too many human lives.

The question we want to answer is: If we isolate $Q$ people (from $N$ people in total) how large can we make the fraction $Q / N$ to ensure $\mathcal{R}_{\text {eff }}<1$ after the isolation simply due to herd immunity? 
To answer this question we distinguish between two cases. In the first case, the dynamical divide and conquer strategy, we assume that all infections occur merely due to the dynamics of the SIR-model without any further intervention. In the second case, the divide and conquer strategy with intentional infections, we admit interventions to maximise immunity.

In what follows we are ignorant about all practical problems with these strategies such as strictly isolating millions of people for many months or even more. In this sense this discussion remains rather academic.

\subsection{Dynamical divide and conquer strategy}

Since we isolate $Q$ people initially, we have $S(0) \simeq N-Q$ susceptible people left (assuming $I(0) \ll N)$. The initial effective reproduction number corresponding to the left picture in Figure 3 is

$$
\mathcal{R}_{\mathrm{eff}, 0}=\frac{\beta}{\gamma} S(0) \simeq \frac{\beta}{\gamma}(N-Q) \simeq \mathcal{R}_{0}\left(1-\frac{Q}{N}\right)
$$

Now, one might suggest to isolate sufficiently many people to reach $\mathcal{R}_{\text {eff, } 0}<1$ in the first place. But then the isolation would have to last until effective vaccination is available. Therefore, the divide and conquer strategy relies on herd immunity after releasing the $Q$ people from isolation.

If we wait long enough for the susceptible population to get infected and to recover, we will have approximately

$$
N-(N-Q) \alpha\left(\mathcal{R}_{\text {eff }, 0}\right)
$$

susceptible people on the right hand side of Figure 3. Recall that $\alpha\left(\mathcal{R}_{\text {eff, }, 0}\right)$ is a solution to (2.15).

Hence, the new effective reproduction number becomes

$$
\mathcal{R}_{\mathrm{eff}} \simeq \frac{\beta}{\gamma}\left(N-(N-Q) \alpha\left(\mathcal{R}_{\mathrm{eff}, 0}\right)\right)=\mathcal{R}_{\mathrm{eff}, 0}\left(\frac{N}{N-Q}-\alpha\left(\mathcal{R}_{\mathrm{eff}, 0}\right)\right) .
$$

By imposing $\mathcal{R}_{\text {eff }}<1$ we obtain the condition

$$
\frac{Q}{N}<1-\frac{\mathcal{R}_{\mathrm{eff}, 0}}{1+\mathcal{R}_{\mathrm{eff}, 0} \alpha\left(\mathcal{R}_{\mathrm{eff}, 0}\right)} .
$$

The optimum can be quantified by solving (2.15) numerically. We find that

$$
\left(\frac{Q}{N}\right)_{\max } \simeq 23 \%
$$

which is reached for $\mathcal{R}_{\text {eff, } 0} \simeq 2.15$. The plot of $(Q / N)$ as a function of $\mathcal{R}_{\text {eff, } 0}$ is shown in Figure 4.

This result is a problem, because the HRG is certainly larger than $23 \%$. For instance, the fraction of people older than 60 years is in Germany above 25\%, wo are included in the HRG if we follow the definition of [9]. Note that in [8] and [10] people are counted to the HRG if they are older than 65 and 70 years, respectively. However, also pregnant women, smokers, chronically ill or obese people, etc. count to the HRG as well. An investigation into estimating the size of the HRG in Germany is beyond the scope of this note, especially because a precise definition of the criteria of the HRG is still under discussion among experts.

Another problem is the peak in the curve of new daily infections among the non-HRG. At $\mathcal{R}_{\text {eff, } 0}=2.15$ one obtains $9.51 \cdot 10^{5}$ infections per day (reached after 5 to 6 months from 


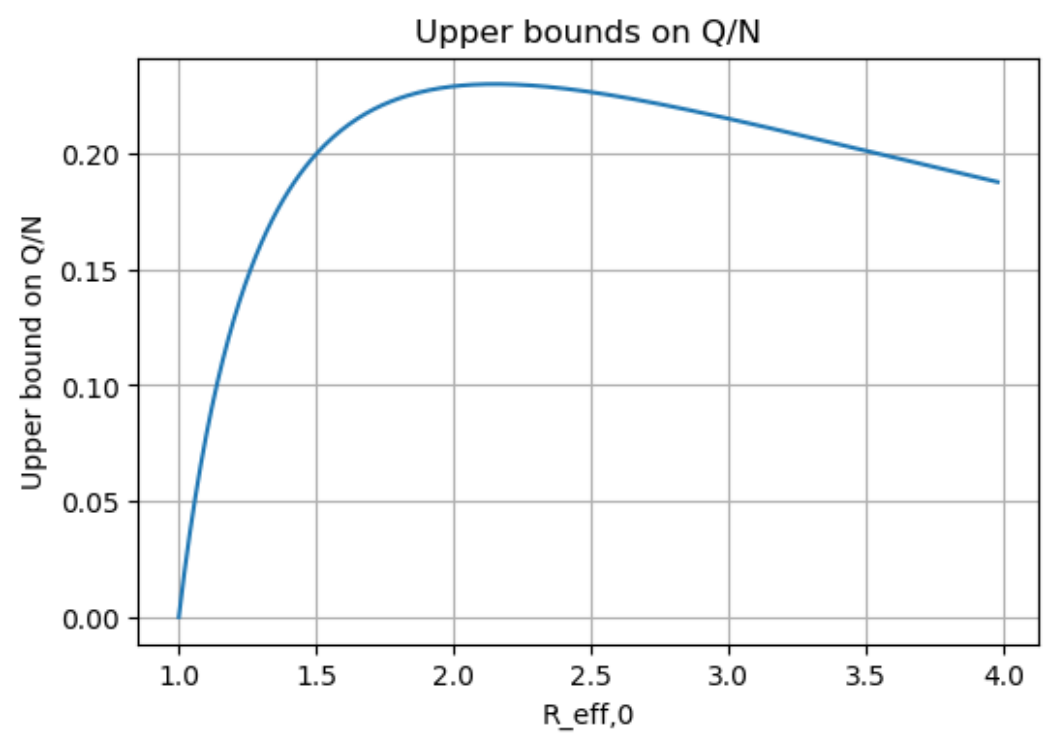

Figure 4: Upper bounds of $Q / N$ as a function of $\mathcal{R}_{\mathrm{eff}, 0}$.

the starting point of divide and conquer). Hence, even if the probability for an individual of the non-HRG to require intensive treatment is ten times smaller than assumed in Section 2.3 the capacities of the health care system are exceeded. To avoid a collapse one rather needs $\mathcal{R}_{\text {eff, } 0} \lesssim 1.8$ (leading to a maximum of $5.8 \cdot 10^{5}$ infections per day). This, however, reduces the maximum of $Q / N$ by only $1 \%$. In general, from Figure 4 it becomes clear that the divide and conquer strategy needs to accept large numbers of new daily infections (see also [1]).

\subsection{Divide and conquer strategy with intentional infections}

There is a loophole that invalidates (3.4) if we admit intentional infections. Thereby one could artificially increase the number of immune people close to $N-Q$. Then,

$$
\mathcal{R}_{\text {eff }}=\frac{\beta}{\gamma} Q=\mathcal{R}_{0} \frac{Q}{N}
$$

or, requiring $\mathcal{R}_{\text {eff }}<1$,

$$
\frac{Q}{N}<\frac{1}{\mathcal{R}_{0}}
$$

Note that in this inequality $\mathcal{R}_{0} \simeq \beta N / \gamma$ is the basic reproduction number for the SARS$\mathrm{CoV}-2$ virus without any particular measures. The result is not surprising given the formula (2.10). Therefore, assuming $\mathcal{R}_{0} \simeq 3$, we get

$$
\left(\frac{Q}{N}\right)_{\max } \simeq 33 \%
$$

This may still turn out to be too small to accommodate the whole HRG among the isolated people.

In any case, even if we assume that we could isolate $50 \%$ of the population (the HRG in the first place), while we ensure that the other $50 \%$ become immune, we lower $\mathcal{R}_{\text {eff }}$ down to $\mathcal{R}_{\text {eff }} \simeq \mathcal{R}_{0} / 2 \sim 1.5$ at the end of isolation. This is the rate at which the initially isolated people 
will get infected. As demonstrated in Section 2.3 this implies a collapse of the health care system, especially because the HRG is more likely to end up in an intensive care unit. Hence, further restrictions would still be unavoidable. Therefore, the divide and conquer strategy seems to provide no reasonable solution.

\subsection{Equipartition strategy?}

Finally we address some interesting effect that occurs upon dividing the population into $n$ equal, non-interacting, groups (see [17]).

First, suppose that the population is split into $n$ pairwise isolated groups such that

$$
S=\sum_{k=1}^{n} S_{k}, \quad I=\sum_{k=1}^{n} I_{k}, \quad R=\sum_{k=1}^{n} R_{k}
$$

Each group individually obeys the differential equations of the SIR-model:

$$
\begin{aligned}
\dot{S}_{k} & =-\beta_{k} S_{k} I_{k}, \\
\dot{I}_{k} & =\beta_{k} S_{k} I_{k}-\gamma I_{k}, \\
\dot{R}_{k} & =\gamma I_{k} .
\end{aligned}
$$

We assume that only the recovery rate $\gamma$ is equal among all groups.

Let us now assume an equipartition, i.e. $S_{k}=S / n, I_{k}=I / n$ and $R_{k}=R / n$. The first differential equation then becomes

$$
\dot{S}=-\frac{\beta_{k}}{n} S I
$$

and the overall transmission rate is reduced to $\beta=\beta_{k} / n$ relative to each group (this observation was made in [17] in a more general model). Unfortunately, this effect is undone in the reproduction rates. We have

$$
\mathcal{R}_{k}(t)=\frac{\beta_{k}}{\gamma} S_{k}=\frac{n \beta}{\gamma} \cdot \frac{S}{n}=\frac{\beta}{\gamma} S=\mathcal{R}(t)
$$

Since it is the reproduction number that determines the dynamics in the SIR-model, we cannot gain any advantage of equipartition in the SIR-model. However, divide and conquer strategies in this spirit may be interesting when taking into account diffusion terms (see e.g. [14]).

\section{The importance of the reproduction number and the present situation in Germany}

So far it has become evident how essential the (effective) reproduction number is in order to control a pandemic. The importance of this factor can be generalised to models beyond the SIR-model, see e.g. [16] for an elaborate model in which $\mathcal{R}(t)$ is systematically determined.

We would like to summarise various arguments why $\mathcal{R}_{\text {eff }}$ (and $\mathcal{R}_{0}$ in particular) is the main parameter determining the next months (possibly more) of our lives:

1. The sign of the term $\mathcal{R}_{\text {eff }}-1$ roughly distinguishes between exponential growth or exponential decay of the number of active infections.

2. $\mathcal{R}_{0}$ determines the required fraction of the population to reach herd immunity.

3. $\mathcal{R}_{0}$ determines the fraction of people who eventually will have contact with the disease if no mitigation measures are imposed. 
4. $\mathcal{R}_{\text {eff }}$ determines the number of daily new infections, see e.g. (2.12).

5. The strategy flattening the curve requires a fine-tuning of $\mathcal{R}_{\text {eff }}$ with a precision of $<10 \%$, see Section 2.3. This is why this strategy is disfavoured in this note.

6. The duration $\tau$ of the strategy stop the curve can be estimated by the formula

$$
\tau \sim \frac{\chi}{\gamma\left(1-\mathcal{R}_{\mathrm{eff}}\right)}
$$

at constant $\mathcal{R}_{\text {eff }}<1$ with $\chi=\chi\left(\mathcal{R}_{\text {eff }}\right)$ being some number that quantifies how far we are away from being able to track infection chains. At early stages of the pandemic $\chi$ is somewhere between $1<\chi<3$, also depending on the effective reproduction number.

7. Taking into account diffusion effects one can show that for $\mathcal{R}_{\text {eff }}>1$ the disease propagates at a speed of $[13 ; 14]$

$$
c \sim \sqrt{1-\frac{1}{\mathcal{R}_{\mathrm{eff}}}}
$$

A large reproduction number implies a large diffusion effect. For $\mathcal{R}_{\text {eff }}<1$ dangerous diffusion effects can be suppressed. Note that diffusion effects may easily trigger a second wave after relaxation of lockdown measures. ${ }^{2}$

In the case of Germany we have 2,8 $\cdot 10^{4}$ known active infections as of 2020-05-03 [35]. If our goal is to reduce the number of new infections to a few hundred per day one obtains $\chi \simeq 2-3$ (as long as the reproduction number is not close to zero), assuming exponential decay of the number of active infections. Keeping $\mathcal{R}_{\text {eff }}<1$ we can therefore estimate the duration of the lockdown to be

$$
\tau \sim \frac{2 \text { weeks }}{1-\mathcal{R}_{\mathrm{eff}}} \cdot \chi\left(\mathcal{R}_{\mathrm{eff}}\right)
$$

Based on [16] a realistic estimate could be $\mathcal{R}_{\text {eff }} \simeq 0.6$ on average during the lockdown. Then we obtain $\tau \sim 12$ weeks from now. If we were able to achieve and keep $\mathcal{R}_{\text {eff }} \simeq 0.4$ then we need restrictions for $\tau \sim 7$ more weeks, and for $\mathcal{R}_{\text {eff }} \simeq 0.2$, in which $\chi \simeq 1.4$ we even have $\tau \sim 3-4$ weeks. Afterwards, when relaxing the restrictions, it will be crucial to track the origins of every single new infection case, hoping that this tracking helps to keep $\mathcal{R}_{\text {eff }}<1$ without further lockdowns. It is essential to be well prepared for this highly non-trivial task.

Finally we would like to stress that any strategy following $\mathcal{R}_{\text {eff }} \simeq 1$ will last for an extremely long time as one can see from (4.3). This is why it is really important to keep the reproduction number significantly below the critical value of 1 in the classical stop the curve strategy.

\section{Conclusion}

In this note we have demonstrated the basic mechanics of pandemics using the SIR-model. Despite its simplicity it captures most of the relevant challenges we are facing in the Covid-19 pandemics. Therefore, we expect that most of the results are also implied by more sophisticated models.

In a short review of the SIR-model applied to the Covid-19 pandemics we have seen that the strategy flattening the curve suffers from a necessary fine-tuning of the effective reproduction

\footnotetext{
${ }^{2}$ This has been pointed out several times by Christian Drosten in his podcast Das Coronavirus-Update at NDR.
} 
number as well as a precise estimation of the capacity of health care systems. This strategy is therefore risky and problematic.

In the main part of this note we approached a divide and conquer strategy. We concluded that the idea to isolate just the high risk group instead of having collective lockdowns fails for two reasons. First, the high risk group is most likely too large to develop herd immunity with respect to the whole population. Second, despite exposing only rather young and healthy people to the virus, the number of daily infections to be expected will probably still exceed Germany's health care system. Although the result on this strategy is negative, we find the problem of quantifying this scenario interesting in itself, resulting in nice inequalities (3.4) and (3.7) with useful applications in the development of new containment strategies.

Finally, we gave several reasons why the (effective) reproduction number is essential when it comes to control the pandemic. Therefore, a monitoring of this parameter is and remains necessary. This, in turn, requires systematic testing. In the case of Germany we estimated that our present restrictions are presumably necessary for a few more months until we are in the position to track the origins of individual infections. A precise exit strategy involves both the size of the reproduction number and the number of new infections each day. The duration until the exit can then be estimated by (4.3). This formula is not meant to be exact but it provides a rough perspective for the next months. The more we can suppress the reproduction number towards zero the earlier we may be able live with far less restrictions.

\section{Acknowledgement}

This note has been written out of private interest in this topic. Hence, no funding is to be reported.

\section{References}

[1] D. Gershon, A. Lipton, and H. Levine, "Managing covid-19 pandemic without destructing the economy," arXiv:2004.10324 [q-bio.PE].

[2] L. Bellomarini, M. Benedetti, A. Gentili, R. Laurendi, D. Magnanimi, A. Muci, and E. Sallinger, "Covid-19 and company knowledge graphs: Assessing golden powers and economic impact of selective lockdown via ai reasoning," arXiv:2004.10119 [cs.AI].

[3] S. M. Iacus, F. Natale, C. Satamaria, S. Spyratos, and M. Vespe, "Estimating and projecting air passenger traffic during the covid-19 coronavirus outbreak and its socio-economic impact," arXiv:2004.08460 [stat.AP].

[4] H. Inoue and Y. Todo, "The propagation of the economic impact through supply chains: The case of a mega-city lockdown against the spread of covid-19," arXiv:2003.14002 [cs.SI].

[5] V. Stojkoski, Z. Utkovski, P. Jolakoski, D. Tevdovski, and L. Kocarev, "The socio-economic determinants of the coronavirus disease (covid-19) pandemic," arXiv:2004.07947 [physics.soc-ph].

[6] F. Liu, A. Page, S. A. Strode, Y. Yoshida, S. Choi, B. Zheng, L. N. Lamsal, C. Li, N. A. Krotkov, H. Eskes, R. van der A, P. Veefkind, P. Levelt, J. Joiner, and O. P. Hauser, "Abrupt declines in tropospheric nitrogen dioxide over china after the outbreak of covid-19," arXiv:2004.06542 [physics.ao-ph]. 
[7] A. A. Toda, "Susceptible-infected-recovered (sir) dynamics of covid-19 and economic impact," arXiv:2003.11221 [q-bio.PE].

[8] "Groups at higher risk for severe illness." https://www.cdc.gov/coronavirus/ 2019-ncov/need-extra-precautions/groups-at-higher-risk.html. Accessed: 2020-05-03.

[9] "People at higher risk from coronavirus." https://www2.hse.ie/conditions/ coronavirus/at-risk-groups .html\#at-risk. Accessed: 2020-05-03.

[10] "Who's at higher risk from coronavirus." https://www.nhs.uk/conditions/ coronavirus-covid-19/people-at-higher-risk-from-coronavirus/ whos-at-higher-risk-from-coronavirus/. Accessed: 2020-05-03.

[11] W. O. Kermack and A. G. McKendrick, "A Contribution to the Mathematical Theory of Epidemics," Proceedings of the Royal Society of London Series A 115 no. 772, (Aug., 1927) $700-721$.

[12] J. A. R. Elizabeth S. Allman, Mathematical models in biology. An introduction. Cambridge University Press, 1 ed., 2003.

[13] J. D. Murray, Mathematical Biology I: An Introduction. Interdisciplinary Applied Mathematics. Springer, 3rd ed., 2007.

[14] J. Murray, Mathematical Biology II Spatial Models and Biomedical Applications. Springer, 3rd ed., 2003.

[15] N. M. Ferguson et al., "Report 9: Impact of non-pharmaceutical interventions (npis) to reduce covid-19 mortality and healthcare demand." https://www. imperial.ac.uk/media/imperial-college/medicine/sph/ide/gida-fellowships/ Imperial-College-COVID19-NPI-modelling-16-03-2020.pdf. Imperial College COVID-19 Response Team; Accessed: 2020-05-03.

[16] S. Khailaie et al., "Estimate of the development of the epidemic reproduction number Rt from Coronavirus SARS-CoV-2 case data and implications for political measures based on prognostics," medRxiv (April 2020) .

[17] G. Oliveira, "Refined compartmental models, asymptomatic carriers and covid-19,". http://dx.doi.org/10.1101/2020.04.14.20065128.

[18] J. Dolbeault and G. Turinici, "Heterogeneous social interactions and the covid-19 lockdown outcome in a multi-group seir model," arXiv:2005.00049 [q-bio.PE].

[19] D. Kai, G.-P. Goldstein, A. Morgunov, V. Nangalia, and A. Rotkirch, "Universal masking is urgent in the covid-19 pandemic: Seir and agent based models, empirical validation, policy recommendations," arXiv:2004.13553 [physics.soc-ph].

[20] R. Goel and R. Sharma, "Mobility based sir model for pandemics - with case study of covid-19," arXiv:2004.13015 [cs.SI].

[21] F. Ndairou, I. Area, J. J. Nieto, and D. F. Torres, "Mathematical modeling of covid-19 transmission dynamics with a case study of wuhan," Chaos, Solitons and Fractals 135 (Jun, 2020) 109846. http://dx.doi.org/10.1016/j.chaos.2020.109846.

[22] C. Bandt, "Transparent covid-19 prediction," arXiv:2004.04732 [q-bio.PE]. 
[23] H. M. Singer, "The covid-19 pandemic: growth patterns, power law scaling, and saturation," arXiv:2004.03859 [q-bio.PE].

[24] J. M. Carcione, J. E. Santos, C. Bagaini, and J. Ba, "A simulation of a covid-19 epidemic based on a deterministic seir model," arXiv:2004.03575 [q-bio.PE].

[25] G. C. Calafiore, C. Novara, and C. Possieri, "A modified sir model for the covid-19 contagion in italy," arXiv:2003.14391 [physics.soc-ph].

[26] J. E. Amaro, "The d model for deaths by covid-19," arXiv:2003.13747 [q-bio.PE].

[27] L. Dell'Anna, "Solvable delay model for epidemic spreading: the case of covid-19 in italy," arXiv:2003.13571 [q-bio.PE].

[28] L. Squillante, I. F. Mello, A. C. Seridonio, and M. de Souza, "Attacking the covid19 with the ising-model and the fermi-dirac distribution function," arXiv:2003.11860 [q-bio.PE].

[29] Q. Li et al., "Early Transmission Dynamics in Wuhan, China, of Novel CoronavirusInfected Pneumonia," The New England Journal of Medicine 382 no. 13, (January, 2020) 1199-1207.

[30] C. L. A. Julien Riou, "Pattern of early human-to-human transmission of Wuhan 2019 novel coronavirus (2019-nCoV), December 2019 to January 2020," Eurosurveillance 25 no. $4,(2020)$.

[31] J. T. Wu et al., "Estimating clinical severity of COVID-19 from the transmission dynamics in Wuhan, China," Nature Medicine 26 no. 5, (March, 2020) 506-510.

[32] S. Sanche et al., "High Contagiousness and Rapid Spread of Severe Acute Respiratory Syndrome Coronavirus 2," Emerging Infectious Diseases 26 no. 7, (April, 2020) 506-510.

[33] A. J. Stier, M. G. Berman, and L. M. A. Bettencourt, "Covid-19 attack rate increases with city size," arXiv:2003.10376 [q-bio.PE].

[34] A. Notari, "Temperature dependence of covid-19 transmission," arXiv:2003.12417 [q-bio.PE].

[35] "Covid-19 data repository by the center for systems science and engineering (csse) at johns hopkins university." https://github.com/CSSEGISandData/COVID-19. Accessed: 2020-05-03.

[36] M. D'Orazio, G. Bernardini, and E. Quagliarini, "How to restart? an agent-based simulation model towards the definition of strategies for covid-19 "second phase" in public buildings," arXiv:2004.12927 [physics.soc-ph].

[37] J.-T. Brethouwer, A. van de Rijt, R. Lindelauf, and R. Fokkink, ""stay nearby or get checked": A covid-19 lockdown exit strategy," arXiv:2004.06891 [cs.SI].

[38] M. Müller, P. M. Derlet, C. Mudry, and G. Aeppli, "Using random testing to manage a safe exit from the covid-19 lockdown," arXiv:2004.04614 [q-bio.PE].

[39] A. Scala, A. Flori, A. Spelta, E. Brugnoli, M. Cinelli, W. Quattrociocchi, and F. Pammolli, "Time, space and social interactions: Exit mechanisms for the covid-19 epidemics," arXiv:2004.04608 [physics.soc-ph]. 
[40] A. Topirceanu, M. Udrescu, and R. Marculescu, "Centralized and decentralized isolation strategies and their impact on the covid-19 pandemic dynamics," arXiv:2004.04222 [q-bio.PE].

[41] D. Meidan, N. Schulamann, R. Cohen, S. Haber, E. Yaniv, R. Sarid, and B. Barzel, "Alternating quarantine for sustainable mitigation of covid-19," arXiv:2004.01453 [q-bio.PE].

[42] C. Manchein, E. L. Brugnago, R. M. da Silva, C. F. O. Mendes, and M. W. Beims, "Strong correlations between power-law growth of covid-19 in four continents and the inefficiency of soft quarantine strategies," arXiv:2004.00044 [physics.soc-ph].

[43] M. Bin, P. Cheung, E. Crisostomi, P. Ferraro, H. Lhachemi, R. Murray-Smith, C. Myant, T. Parisini, R. Shorten, S. Stein, and L. Stone, "On fast multi-shot covid-19 interventions for post lock-down mitigation," arXiv:2003.09930 [physics.soc-ph].

[44] F. Casella, "Can the covid-19 epidemic be controlled on the basis of daily test reports?," arXiv:2003.06967 [physics.soc-ph].

[45] R. Singh and R. Adhikari, "Age-structured impact of social distancing on the covid-19 epidemic in india," arXiv:2003.12055 [q-bio.PE]. 